\title{
Forecasting Drought Using Modified Empirical Wavelet Transform-ARIMA with Fuzzy C-Means Clustering
}

\author{
Muhammad Akram Shaari, Ruhaidah Samsudin, Ani Shabri Ilman \\ Faculty of Computing, Universiti Teknologi Malaysia, Malaysia
}

\begin{tabular}{|c|c|}
\hline Article Info & ABSTRACT \\
\hline & \multirow{10}{*}{$\begin{array}{l}\text { Drought forecasting is important in preparing for drought and its mitigation } \\
\text { plan. This study focuses on the investigating the performance of Auto } \\
\text { Regressive Integrated Moving Average (ARIMA) and Empirical Wavelet } \\
\text { Transform (EWT)-ARIMA based on clustering analysis in forecasting } \\
\text { drought using Standard Precipitation Index (SPI). Daily rainfall data from } \\
\text { Arau, Perlis from } 1956 \text { to } 2008 \text { was used in this study. SPI data of } 3,6,9,12 \\
\text { and } 24 \text { months were then calculated using the rainfall data. EWT is employed } \\
\text { to decompose the time series into several finite modes. The EWT is used to } \\
\text { create Intrinsic Mode Functions (IMF) which are used to create ARIMA } \\
\text { models. Fuzzy c-means clustering is used on the instantaneous frequency } \\
\text { given by Hilbert Transform of the IMF to create several clusters. The } \\
\text { objective of this study is to compare the effectiveness of the methods in } \\
\text { accurately forecasting drought in Arau, Malaysia. It was found that the } \\
\text { proposed model performed better compared to ARIMA and EWT-ARIMA. } \\
\text { Copyright } \odot 2018 \text { Institute of Advanced Engineering and Science. } \\
\text { All rights reserved. }\end{array}$} \\
\hline Received May, 2, 2018 & \\
\hline Revised Jun 3, 2018 & \\
\hline Accepted Jun 17, 2018 & \\
\hline \multirow{6}{*}{$\begin{array}{l}\text { Keywords: } \\
\text { ARIMA } \\
\text { Drought forecasting } \\
\text { Empirical wavelet transform } \\
\text { Fuzzy c-means clustering } \\
\text { SPI }\end{array}$} & \\
\hline & \\
\hline & \\
\hline & \\
\hline & \\
\hline & \\
\hline \multicolumn{2}{|l|}{ Corresponding Author: } \\
\hline \\
\hline \multicolumn{2}{|l|}{ Faculty of Computing, } \\
\hline \multicolumn{2}{|c|}{ Universiti Teknologi Malaysia, Malaysia. } \\
\hline \multicolumn{2}{|c|}{ Email: ruhaidah@utm.my } \\
\hline
\end{tabular}

\section{INTRODUCTION}

Drought is not unfamiliar and happens across the world. Drought can be destructive to lives of people in an area. Drought is a natural hazard and can be defined as a deficiency in precipitation over an extended period, which is usually a season or more that causes water shortage. Droughts occur in all climatic zone, which are given by the drop of precipitation over a long period of time over a season or a year [1].

Drought forecasting can be done either using physical model or data driven models. Physical models are more complex than data driven models since they require more data as its input, while, data driven models require less information and have rapid development times [2]. They are also found to be accurate enough, thus they are widely used in hydrological forecasting. A number of studies used autoregressive integrated moving average (ARIMA), a stochastic model, to forecast drought [3], such as in [4], [5]. Mossad and Alazba [5] used ARIMA to forecast drought in arid region in Kingdom of Saudi Arabia. Mishra et. al. [4] used a hybrid stochastic and neural network model to forecast drought in Kansabati region in India. Generally, ARIMA was found to be suitable to be used for drought forecasting using standard precipitation index (SPI) time series. In other field, ARIMA was shown to be able to effectively forecast Malaysian gold bullion price [6]. Stochastic methods such as ARIMA are good in forecasting linear time series, however they does not perform as well when forecasting non-linear time series [7].

Wavelet transform are used to improve forecast accuracy, where they are applied along with artificial intelligence models and stochastic models. Wavelet transform is used to decompose the time series into multiple sub series of smooth and detailed component [8]. Wavelets have been shown to increases the forecast accuracy of ARIMA [9], [10] and artificial neural network models [11]. In [10], Kriechbaumer et al. found out that the use of Wavelet transform with ARIMA increases the accuracy of metal price forecast. 
The study investigates numerous combination of mother wavelet and decomposition levels and found out that both variables affects forecasting accuracy. Wavelets separate the seasonal information and the detailed information in a time series, where the linear and non-linear property of the time series is extracted [8].

Multi Resolution Analysis (MRA) have been employed in forecasting using wavelets. The application of MRA in forecasting is as follow, firstly, the time series is decomposed to obtain smooth series and multiple detail series. Next, forecasting method, such as ARIMA is commonly used to extend the subseries. Lastly, the multiple subseries is reconstructed to the original time series [9]. In DWT, this is done by obtaining the summation of each of the subseries. Forecasting using MRA is capable of obtain a more accurate forecasting compared to directly forecasting the original series. This is because the variance of the subseries is more stable and they typically have no outlier [10].

Empirical Mode Decomposition (EMD) is another signal decomposition method that is frequently explored in forecasting. Several studies in wind speed forecasting and annual precipitation forecasting [12]-[14] have shown that forecasting the IMFs of EMD results in more accurate forecasting compared to direct forecasting. EMD have been shown to be able to increase forecast accuracy of Relevance Vector Machine (RVM) [14].

Jerome Gilles [15] proposed empirical wavelet transform (EWT) that identifies the many unalike intrinsic modes of a time series and extracts them. Compared to EMD, EWT gives a more consistent decomposition [15]. Since EWT is conceptually similar to DWT, it was found that using EWT in time series prediction increases the accuracy. EWT has been used in wind speed forecasting along with Gaussian Process Regression (GPR), and it was found out that the models that utilized EWT have higher accuracy compared to the models that does implement EWT [7], [16]. In both studies, EWT was used to denoise the input data.

Several studies have explored on the use of clustering analysis to further improve the forecast accuracy [17]. In [18], IMF from EMD are clustered using k-means clustering on the instantaneous frequency of the IMFs. In [19], [20], Permutation Distribution Clustering (PDC) clustering is employed with EMD and Least Support Square Vector Machine (LSSVM) to forecast exchange rate. From the studies, clustering generally increases the accuracy of the forecasts. Fuzzy c-means clustering has also been implemented in forecasting [21]. However, currently there are no studies done on ensemble forecasting based on c-means clustering.

The objective of this research paper to investigate the performance of ARIMA, EWT ARIMA and modified EWT-ARIMA with fuzzy c-means clustering regarding to their performance in forecasting drought using Standard Precipitation Index (SPI). SPI 3, SPI 6, SPI 9, SPI 12 and SPI 24 were used as indicator for short term and long-term drought. Mean absolute error (MAE) and root mean square error (RMSE) were utilized to measure the forecasting performance. ARIMA is widely used in drought forecasting, however it is not able to accurately forecast drought because ARIMA does not excel at non-linear data, which the SPI data are.

\section{RESEARCH METHOD}

\subsection{Standard Precipitation Index}

The Standard Precipitation Index (SPI) was developed by McKee et al. [22] as a drought indicator which standards the rainfall excess/deficit on temporal and regional basis. To enable quantifying precipitation deficits of multiple time scales, many drought studies used SPI. These time scales show the impact of drought on the availability of the different water resources. Requiring only records of precipitation, SPI enables an analyst to determine the rarity of a drought or a non-typical wet event relative to a particular time scale and can be used worldwide [23]. A drought event occurs during the time when SPI is continuously negative. The drought event ends when the SPI turns positive. Table 1 shows the SPI based drought classification.

Table 1. Drought Classification using SPI

\begin{tabular}{cc}
\hline SPI Value & Category \\
\hline Higher than 2.00 & Wet (Extreme) \\
1.50 until 1.99 & Wet (Severe) \\
1.00 until 1.49 & Wet (Moderate) \\
-0.99 until 0.99 & Near Normal \\
-1.49 until -1.00 & Dry (Moderate) \\
-1.99 until -1.50 & Dry (Severe) \\
Less than -2.00 & Dry (Extreme) \\
\hline
\end{tabular}




\subsubsection{ARIMA}

Scientific applications of time series model have been widespread, however, it has been limited in its application in drought forecasting [3]. The advantages of using time series model are their identification, estimation, and diagnostic check capable of systematic search for model development [24]. One of the commonly-used time series model is Autoregressive Integrated Moving Average (ARIMA). ARIMA is popular due to its statistical properties and its ability to implement various exponential smoothing models [25].

The general non-seasonal ARIMA $(p, d, q)$ model is given by:

$$
\phi_{p}(B) \nabla^{\mathrm{d}} x_{t}=\theta_{q}(B) a_{t}
$$

where $\phi(B)$ and $\theta(B) a_{t}$ are polynomials of order $p$ and $q$, respectively. For seasonal model, the seasonal ARIMA $(p, d, q)(P, D, Q)_{s}$ Is given by:

$$
\phi_{p}(B) \Phi_{P}\left(B^{S}\right) \nabla^{\mathrm{d}} \nabla_{s}^{D} x_{t}=\theta_{q}(B) \Theta_{Q}\left(B^{S}\right) a_{t}
$$

where $P, D, Q$ are the order of seasonal autoregression, number of seasonal differencing, and the order of seasonal MA respectively. Seasonal ARIMA models are used for time series that have cyclic features. In drought forecasting, the data may feature cyclic properties.

\subsection{Empirical Wavelet Transform}

The empirical wavelets can be defined as bandpass filters on each $\Lambda_{n}$, where $\Lambda_{n}$ denotes each segment $\Lambda_{n}=\left[w_{n-1}, w_{n}\right]$ and $\bigcap_{n=1}^{N} \Lambda_{n}=[0, \pi] . \forall_{n}>0$ TheEequation (3) and (4) follows defines the empirical scaling functions and empirical wavelets [7], [15]

$$
\begin{gathered}
\hat{\phi}_{n}(\omega)=\left\{\begin{array}{cc}
1 & \text { if }|\omega| \leq(1-\gamma) \omega_{n} \\
\cos \left[\frac{\pi}{2} \beta\left(\frac{1}{2 \tau_{n}}\left(|\omega|-\omega_{n}+\tau_{n}\right)\right)\right] \text { if }(1-\gamma) \omega_{n} \leq|\omega| \leq(1+\gamma) \omega_{n} \\
0 & \text { otherwise }
\end{array}\right. \\
\hat{\phi}_{n}(\omega)=\left\{\begin{array}{cc}
1 & \text { if }(1+\gamma) \omega_{n} \leq|\omega| \leq(1+\gamma) \omega_{n+1} \\
\cos \left[\frac{\pi}{2} \beta\left(\frac{1}{2 \tau_{n+1}}\left(|\omega|-\omega_{n+1}+\tau_{n+1}\right)\right)\right] & \text { if }(1-\gamma) \omega_{n+1} \leq|\omega| \leq(1-\gamma) \omega_{n+1} \\
\sin \left[\frac{\pi}{2} \beta\left(\frac{1}{2 \tau_{n}}\left(|\omega|-\omega_{n}+\tau_{n}\right)\right)\right] & \text { if }(1+\gamma) \omega_{n} \leq|\omega| \leq(1-\gamma) \omega_{n} \\
0 & \text { otherwise }
\end{array}\right.
\end{gathered}
$$

The function $\beta(x)$ is an arbitrary $C^{k}([0,1])$ function which satisfies the following properties $\beta(x)=\left\{\begin{array}{l}0 \text { if } x \leq 0 \\ 1 \text { if } x \geq 0\end{array}\right.$ and $\beta(x)+\beta(1-x)=1, \forall x \in[0,1]$.

The set $\left\{\phi_{1}(t),\left\{\varphi_{n}(t)\right\}_{n=1}^{N}\right\}$ is a tight frame of $L^{2}(R)$ when $\gamma<\min _{n}\left(\frac{\omega_{n+1}-\omega_{n}}{\omega_{n+1}+\omega_{n}}\right)$. The inner product of the signal and empirical wavelets yields the detail coefficients:

$$
\begin{aligned}
w_{f}^{\epsilon}(n, t)= & \left\langle f, \varphi_{n}\right\rangle=\int f(\tau) \overline{\varphi_{n}(\tau-t)} d \tau \\
& =f(\tau))\left(\hat{\varphi}_{n}(\tau-t)\right)^{\vee}
\end{aligned}
$$

The approximation coefficients are then calculated using the inner products and the scaling function

$$
\begin{aligned}
w_{f}^{\epsilon}(0, t) & =\left\langle f, \phi_{1}\right\rangle=\int f(\tau) \overline{\phi_{1}(\tau-t)} d \tau \\
& =f(\tau))\left(\overline{\hat{\phi}_{1}(\tau-t)}\right)^{\vee}
\end{aligned}
$$

The reconstruction can be obtained: 


$$
\begin{aligned}
& f(t)=w_{f}^{\epsilon}(0, t) * \phi_{1}(t)+\sum_{n=1}^{N} w_{f}^{\epsilon}(n, t) * \varphi_{n}(t) \\
& =\left(\widehat{w_{f}^{\epsilon}}(0, \omega) * \phi_{1}(\omega)+\sum_{n=1}^{N} \widehat{w_{f}^{\epsilon}}(n, \omega) * \varphi_{n}(\omega)\right)^{\vee}
\end{aligned}
$$

Considering this formalism, the empirical mode is given by:

$f_{0}(t)=w_{f}^{\epsilon}(0, t) * \varphi_{1}(t)$

$f_{k}(t)=w_{f}^{\epsilon}(k, t) * \varphi_{k}(t)$

\subsection{EWT-ARIMA}

EWT borrows the framework from wavelet transform [6]. Thus, the application of wavelet transform can also be applied to EWT. Similar to wavelet decomposition, the time series is decomposed to a number of modes using EWT. Figure 1 shows the framework for EWT-ARIMA. The time series is decomposed into several modes using EWT. Numbers of studies have shown that three-level wavelet decomposition produces good forecasting result. Based on the papers that implemented EWT [7], [16], [26], the mode that is commonly used for EWT is 3.

However, in [27], the author of EWT proposed a method to determine the number of modes for the decomposition. This method separates histograms into two classes, where the goal is to find the threshold $T$ such that the intra-variance of the class is mimimal while the inter class variance is maximum. This study uses this method to determine the number of modes decomposed using EWT. The following are the process for performing EWT [15]:

1. Perform pre-processing to detect peak.

2. Perform spectrum separation based on the detected maxima.

3. Construct corresponding wavelet filter bank.

4. Construct the wavelets based on the wavelet filter bank.

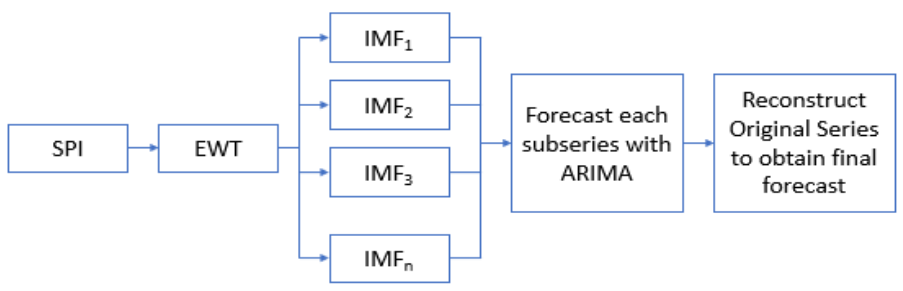

Figure 1. Overall workflow for EWT-ARIMA

\subsection{Hilbert Transform}

Hilbert transform is defined as following:

$$
\tilde{x}(t)=\frac{p}{\pi} \int \frac{x\left(t^{\prime}\right)}{t-t^{\prime}} d t^{\prime}
$$

Where $\mathrm{p}$ is the Cauchy principal value. The instantaneous frequency $\omega(t)$ thus can be calculated using $\omega(t)=\frac{1}{2 \pi} \frac{d \varphi(t)}{d t}$, where $\varphi(t)=\arctan \frac{\tilde{s}(t)}{s(t)}$.

\subsection{Fuzzy C-means Clustering}

Fuzzy c-means(FCM) clustering is a clustering algorithm that determines the degree of membership of a data with regards to its cluster or class. The objective function of FCM algorithm is as follow and it is computed using the membership value and Euclidian distance. 
$J_{m}(W, P)=\sum_{\substack{1 \leq k \leq n \\ 0 \leq i \leq c}}\left(w_{i k}\right)^{m}\left(d_{i k}\right)^{2}$

where $\left(d_{i k}\right)=\left\|x_{k}-p_{i}\right\|$

Minimizing the objective function, thus the FCM membership function is as follows [28]:

$$
\mu_{i j}=\left[\sum_{t=1}^{c}\left(\frac{\left\|x_{j}-v_{i}\right\|_{A}}{\left\|x_{j}-v_{t}\right\|_{A}}\right)^{\frac{2}{m-1}}\right]^{-1}
$$

where $\mu_{i j}$ is th membership value of $j$ th sample and $i$ th cluster, $c$ is the number o cluster, $x_{j}$ is the $j$ th sample and $v_{i}$ cluster center of the $i$ th cluster. \|\|$_{A}$ is the norm function.

\subsection{EWT-ARIMA Clustering Analysis}

The overall framework for the proposed method is as in Figure 1. For both methods, firstly the SPI data is decomposed into several modes using EWT. Next, IMFs are constructed each of the modes from EWT. Hilbert transform are done on each of the IMFs to obtain its instantaneous frequency, where they will be clustered using fuzzy c-means clustering. From the obtained clusters, 3 subseries are created by summing the IMFs according tho the cluster information. Then, the high, medium, and low frequency series are fitted with ARIMA models. Forecast values are obtained for each of the 3 subseries. Lastly, for both methods, the forecasting results are combined to obtain the final forecasting result. Figure 2 shows the overall process for EWT-ARIMA Clustering analysis.

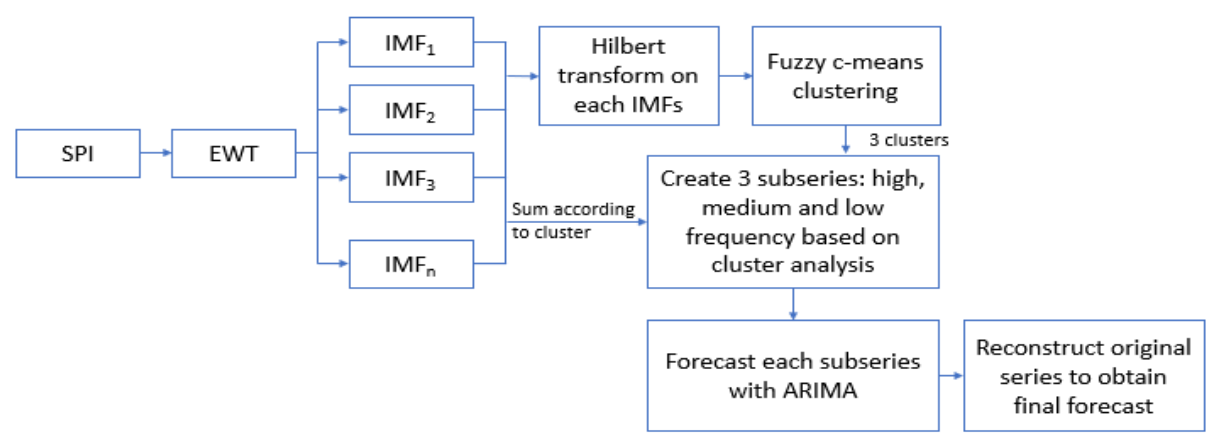

Figure 2. Overall workflow for modified EWT-ARIMA

\subsection{Performance Comparison}

The performance of each method is evaluated by comparing the error statistics. The error statistics used were MAE and RMSE. They are given by:

$$
\begin{aligned}
& \mathrm{MAE}=\frac{1}{N} \sum_{i=1}^{N}\left|\mathrm{EST}_{i}-\mathrm{OBS}_{i}\right| \\
& \mathrm{RMSE}=\sqrt{\frac{1}{N} \sum_{i=1}^{N}\left(\mathrm{EST}_{i}-\mathrm{OBS}_{i}\right)^{2}}
\end{aligned}
$$

Where $\mathrm{EST}_{i}$ and $\mathrm{OBS}_{i}$ denote $i$ th estimated and observed values respectively. The lower the value of error, the better is the performance of the forecasting model.

\subsection{Study Area}

The physical area covered in this study is the area around Arau, Perlis. The data consist of daily rainfall value from 1956 to 2008 . 
Table 2. Statistical Description of Data

\begin{tabular}{ccccc}
\hline & Mean & St. dev. & Min. & Max. \\
\hline Rainfall & 5.459 & 12.598 & 0.000 & 180.300 \\
\hline
\end{tabular}

\section{RESULTS AND ANALYSIS}

In this section, it is explained the results of research and at the same time is given the comprehensive discussion. Results can be presented in figures, graphs, tables and others that make the reader understand easily [2], [5]. The discussion can be made in several sub-chapters.

\subsection{ARIMA}

For ARIMA, there are three stages in time series model development, namely: identification, estimation, and diagnostic check [29]. For each of the SPI time scale, data set from 1956 to 1998 is applied in the estimation of the model parameters. Data from 1999 to 2008 is used to determine the accuracy of the forecast. SPI 3, 6, 9, 12 and 24 are used in this study. SPI is calculated from the rainfall data. Figure 3 shows the data consisting of SPI 3, 6 and 12 .

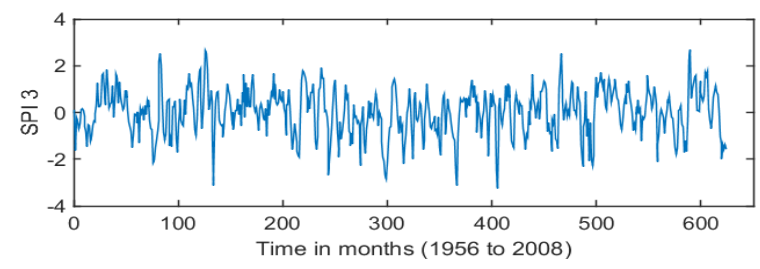

Figure 3. SPI 3 data over from 1956 to 2008

Figure 2 shows the autocorrelation function (ACF) and partial autocorrelation function (PACF) used of SPI 3. From the ACF and PACF plot, value suitable for ARIMA model obtained are $p=1,2,3,4$, $q=0,1,2, P=1,2$, and $Q=0$. The combination of models suitable are obtained from the value and the fitness of the models determined.
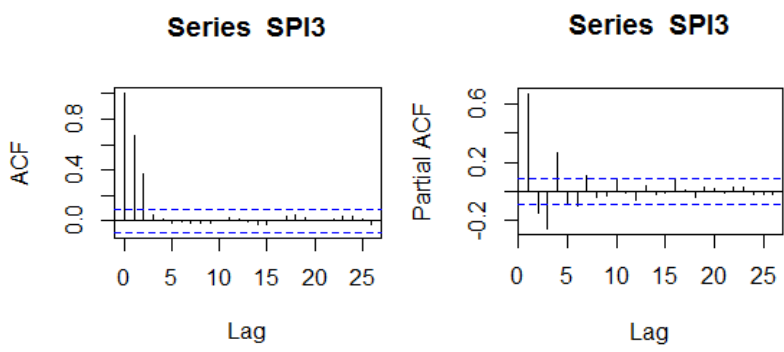

Figure 4. ACF and PACF of SPI 3

To select the best fit model, Akaike Information Criterion (AIC) is used. The best fit model is selected from the model with the lowest AIC. The mathematical formula for AIC is defined as [30]:

$$
A I C=-2 \log L+2 m
$$

where $m=(p+q+P+Q)$ is the number of terms estimated in the model and L signifies the likelihood of the ARIMA models and it is monotonically decreasing function of the sum of squared residuals. The best fit model for SPI 3 obtained is $(2,0,2)$. 


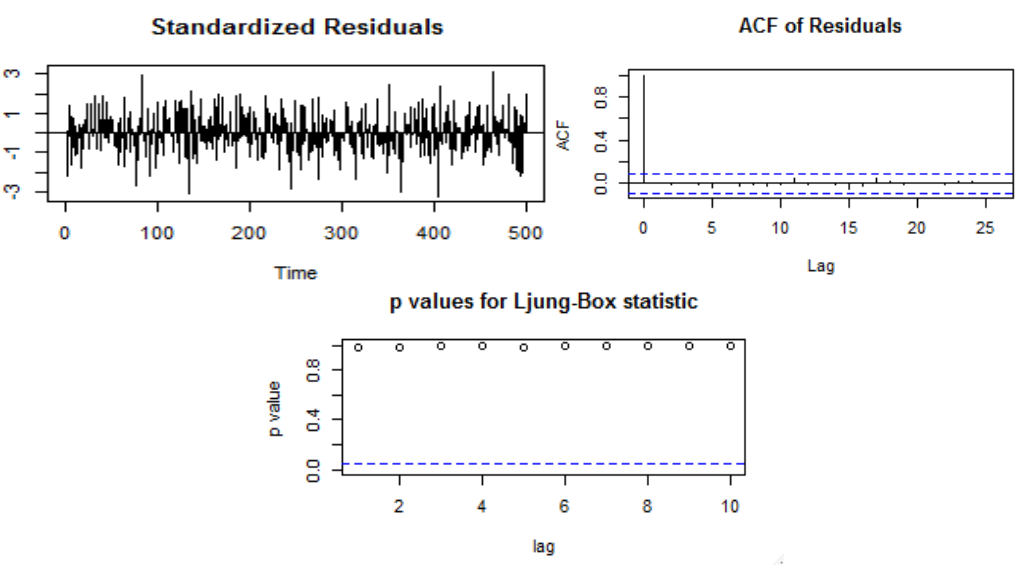

Figure 5. Diagnostic Checking of ARIMA

To verify that a model is adequate, diagnostic check is performed among the identified models. This includes the study of residuals to see if there are patterns unaccounted for. After fitting the model, the residual left over should be white noise for a model to be considered as a good model. The residual ACF is used to determine whether the residuals are white noise. Residuals that are largely different from zero give an indication that the model may be inadequate. Figure 5 shows the diagnostic check of the ARIMA model obtained for SPI 3.

\subsection{EWT-ARIMA}

EWT is used to decompose the SPI data into several IMF. The number of IMF depends on the data, where it is determined using Otsu's method [27]. Table 3 shows the number of IMFs generated by EWT process. Figure 6 shows SPI 12 decomposed to 6 IMFs using EWT. ARIMA model is developed for each of the subseries, using auto ARIMA function found in R, and it is used to perform the forecast. The result of the forecast on each of the subseries is obtained, which is then used to reconstruct the original time series. The result of the reconstruction is the forecast result of EWT-ARIMA.

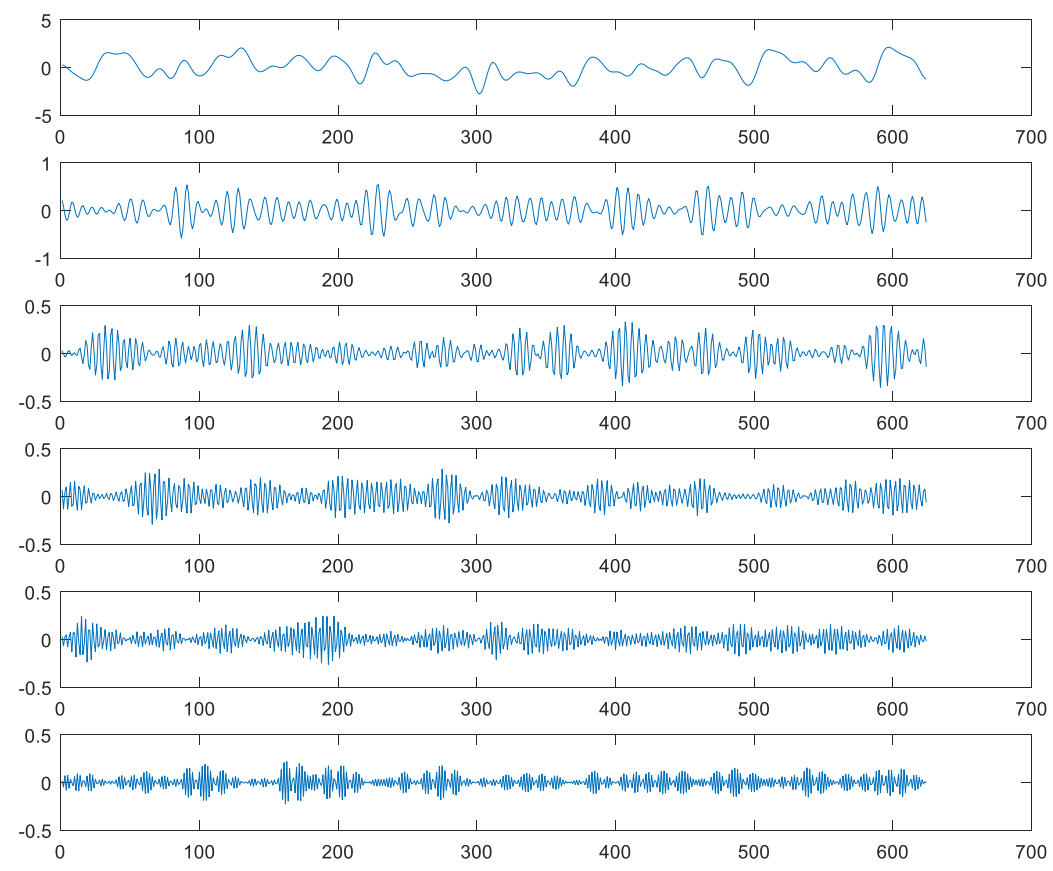

Figure 6. EWT decomposition of SPI 12 
Table 3. Number of IMFs produced by EWT

\begin{tabular}{cccccc}
\hline SPI & SPI 3 & SPI 6 & SPI 9 & SPI 12 & SPI 24 \\
\hline Number of IMFs & 12 & 6 & 5 & 6 & 12 \\
\hline
\end{tabular}

\subsection{EWT-ARIMA Clustering}

Fuzzy c-means clustering is used to cluster the IMF created from EWT into a certain number of cluster. The number of cluseter chosen is 3 . Several studies have used 3 cluster [17], [18]. Figure 7 shows the combined into 3 wavelets based on the result of FCM clustering. IMF 1, 2 is combined to form the low frequency compoment. IMF 3,4,5 is combined to form the medium frequency component while IMF 6 is used as the high frequency component. Table 4 shows the details of the components for each cluster. From the components, ARIMA is used to perform forecasts.
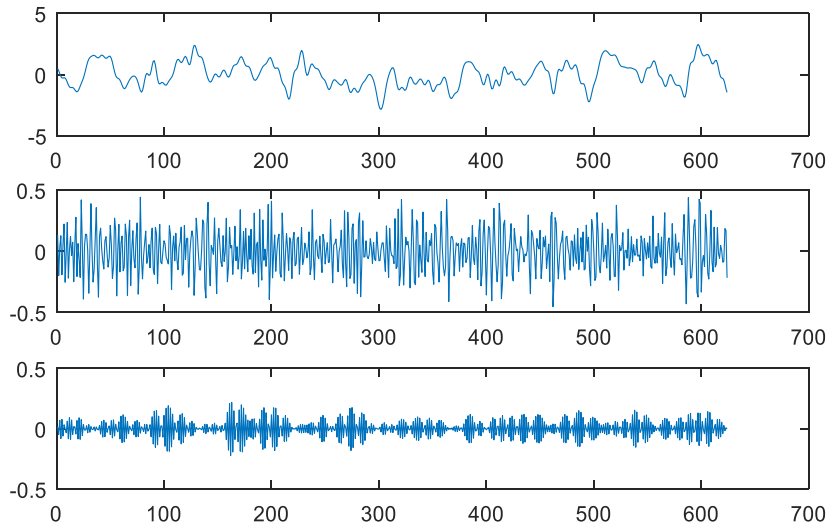

Figure 7. 3 cluster from EWT decomposition on SPI 12

Table 4. Components for Each Clusters

\begin{tabular}{|c|c|c|}
\hline SPI & \multicolumn{2}{|c|}{ Cluster Components } \\
\hline \multirow[t]{3}{*}{ SPI 3} & $\mathrm{G} 1=$ & $\{$ IMF1-IMF5 $\}$ \\
\hline & $\mathrm{G} 2=$ & $\{$ IMF6-IMF9\} \\
\hline & $\mathrm{G} 3=$ & $\{\mathrm{IMF} 10-\mathrm{IMF} 12\}$ \\
\hline \multirow[t]{3}{*}{ SPI 6} & $\mathrm{G} 1=$ & $\{\mathrm{IMF} 1-\mathrm{IMF} 2\}$ \\
\hline & $\mathrm{G} 2=$ & $\{$ IMF3-IMF4 $\}$ \\
\hline & $\mathrm{G} 3=$ & $\{$ IMF5-IMF6 $\}$ \\
\hline \multirow[t]{3}{*}{ SPI 9} & $\mathrm{G} 1=$ & $\{$ IMF1-IMF2 $\}$ \\
\hline & $\mathrm{G} 2=$ & $\{$ IMF3-IMF4 $\}$ \\
\hline & $\mathrm{G} 3=$ & $\{$ IMF5 $\}$ \\
\hline SPI & $\mathrm{G} 1=$ & $\{$ IMF1-IMF2 $\}$ \\
\hline \multirow[t]{2}{*}{12} & $\mathrm{G} 2=$ & $\{$ IMF3-IMF5 $\}$ \\
\hline & $\mathrm{G} 3=$ & $\{$ IMF6 $\}$ \\
\hline SPI & $\mathrm{G} 1=$ & $\{\mathrm{IMF} 1-\mathrm{IMF} 4\}$ \\
\hline \multirow[t]{2}{*}{24} & $\mathrm{G} 2=$ & $\{$ IMF5-IMF8 $\}$ \\
\hline & $\mathrm{G} 3=$ & $\{$ IMF9-IMF12\} \\
\hline
\end{tabular}

\subsection{Performance Evaluation}

The result of the study is obtained as in Table 5. MAE and RMSE is calculated from the forecasting results of each models and are compared. Table 5 shows the MAE and RMSE for testing data of the models on each SPI. Lower values of MAE and RMSE indicates a higher forecast accuracy.

EWT-ARIMA performed better compared to ARIMA on all SPI. This is due to the nonlinear nature of SPI data, which cannot be forecasted accurately using a linear method such as ARIMA. EWT separates the linear and nonlinear component of the data, thus ARIMA is able to forecast them with greater accuracy. This show that EWT is suitable to decompose the non-linear nature of SPI data.

The proposed model performed better compared to ARIMA and EWT-ARIMA on all SPI except SPI 9 and 3. This indicates that the proposed model is suitable to be used for long term drought forecasting. Comparing ARIMA, EWT-ARIMA and modified EWT-ARIMA, it is generally safe to assume the proposed 
modified EWT-ARIMA performs the best, since it preformed better on 3 out of the 5 SPI tested. The result agrees with previous reseach that has proven that clustering on IMFs from EMD improves the forecast accuracy [18]-[20]. Implementation of fuzzy c-means clustering improves the forecast accuracy by grouping the IMFs according to its structure based on the intrinsic frequency of the IMFs.

Table 5. MAE and RMSE of the Models

\begin{tabular}{ccccccc}
\hline Models & \multicolumn{2}{c}{ ARIMA } & \multicolumn{2}{c}{ EWT-ARIMA } & \multicolumn{2}{c}{ EWT-ARIMA Clustering } \\
\hline SPI & MAE & RMSE & MAE & RMSE & MAE & RMSE \\
SPI 3 & 0.4361 & 0.5619 & 0.3655 & 0.4764 & 0.3834 & 0.4982 \\
SPI 6 & 0.2979 & 0.3973 & 0.2744 & 0.3512 & 0.1068 & 0.1397 \\
SPI 9 & 0.2472 & 0.3250 & 0.1165 & 0.1440 & 0.1275 & 0.1621 \\
SPI 12 & 0.2230 & 0.2919 & 0.1570 & 0.2021 & 0.0703 & 0.0891 \\
SPI 24 & 0.1557 & 0.2008 & 0.1446 & 0.1857 & 0.0586 & 0.0732 \\
\hline
\end{tabular}

\section{CONCLUSION}

This research compares ARIMA and EWT ARIMA and EWT-ARIMA clustering. SPI is used as the drought index and SPI 3, 6, 9, 12 and 24 was calculated using rainfall data from Arau, Perlis. ARIMA model was developed using Box-Jenkins methodology for each SPI. This research uses MAE and RMSE to compare the effectiveness of the prediction from the studied models. It was found that EWT-ARIMA clustering performed better compared to ARIMA and EWT-ARIMA on SPI 6, 12, and 24. EWT-ARIMA performed better compared to EWT-ARIMA Clustering on SPI 3 and 9.

\section{ACKNOWLEDGEMENTS}

High appreciation is given to Department of Irrigation and Drainage, Ministry of Natural Resources and Environment, Malaysia for providing the rainfall data to complete the research. Finally, thanks are given to Universiti Teknologi Malaysia (UTM). This work was supported by the Ministry of Higher Education and Ministry of Science, Technology and Innovation (MOSTI) under Grant Vot 4F875.

\section{REFERENCES}

[1] A. K. Mishra and V. P. Singh, "A review of drought concepts,” J. Hydrol., vol. 391, no. 1-2, pp. 202-216, Sep. 2010.

[2] A. Belayneh, J. Adamowski, B. Khalil, and B. Ozga-Zielinski, "Long-term SPI drought forecasting in the Awash River Basin in Ethiopia using wavelet neural network and wavelet support vector regression models," J. Hydrol., vol. 508, pp. 418-429, Jan. 2014.

[3] A. K. Mishra and V. P. Singh, "Drought modeling - A review," J. Hydrol., vol. 403, no. 1-2, pp. 157-175, Jun. 2011.

[4] A. Mishra, V. Desai, and V. Singh, "Drought forecasting using a hybrid stochastic and neural network model," $J$. Hydrol. Eng., vol. 12, no. 6, pp. 626-638, 2007.

[5] A. Mossad and A. A. Alazba, "Drought Forecasting Using Stochastic Models in a Hyper-Arid Climate," Atmosphere, vol. 6, no. 4, pp. 410-430, Apr. 2015.

[6] L. Abdullah, "ARIMA model for gold bullion coin selling prices forecasting," Int. J. Adv. Appl. Sci., vol. 1, no. 4, pp. 153-158, 2012.

[7] J. Hu and J. Wang, "Short-term wind speed prediction using empirical wavelet transform and Gaussian process regression," Energy, vol. 93, pp. 1456-1466, Dec. 2015.

[8] V. Nourani, A. Hosseini Baghanam, J. Adamowski, and O. Kisi, "Applications of hybrid wavelet-Artificial Intelligence models in hydrology: A review," J. Hydrol., vol. 514, pp. 358-377, Jun. 2014.

[9] S. Schlüter and C. Deuschle, "Using Wavelets for Time Series Forecasting - Does it Pay Off?," 2010.

[10] T. Kriechbaumer, A. Angus, D. Parsons, and M. Rivas Casado, "An improved wavelet-ARIMA approach for forecasting metal prices," Resour. Policy, vol. 39, pp. 32-41, Mar. 2014.

[11] R. V. Ramana, B. Krishna, S. R. Kumar, and N. G. Pandey, "Monthly Rainfall Prediction Using Wavelet Neural Network Analysis," Water Resour. Manag., vol. 27, no. 10, pp. 3697-3711, Aug. 2013.

[12] Y. P. Liu, Y. Wang, and Z. Wang, "RBF Prediction Model Based on EMD for Forecasting GPS Precipitable Water Vapor and Annual Precipitation,” Adv. Mater. Res., vol. 765-767, pp. 2830-2834, 2013.

[13] Z. Guo, W. Zhao, H. Lu, and J. Wang, "Multi-step forecasting for wind speed using a modified EMD-based artificial neural network model,” Renew. Energy, vol. 37, no. 1, pp. 241-249, Jan. 2012.

[14] Y. Y. U. Guang, Z. Y. U. Hu, and X. S. I. of B. and T. Liu, "A Novel Strategy for Wind Speed Prediction in Wind Farm,” TELKOMNIKA Indones. J. Electr. Eng., vol. 11, no. 12, pp. 7007-7013, 2013.

[15] J. Gilles, "Empirical Wavelet Transform," IEEE Trans. Signal Process., vol. 61, no. 16, pp. 3999-4010, Aug. 2013. 
[16] J. Hu, J. Wang, and K. Ma, “A hybrid technique for short-term wind speed prediction,” Energy, vol. 81, pp. 563574, Mar. 2015.

[17] S. Aghabozorgi, A. Seyed Shirkhorshidi, and T. Ying Wah, "Time-series clustering - A decade review," Inf. Syst., vol. 53, no. Supplement C, pp. 16-38, Oct. 2015.

[18] A. Shabri, "A modified EMD-ARIMA based on clustering analysis for fishery landing forecasting," vol. 10, pp. 1719-1729, Jan. 2016.

[19] N. I. A. Rashid, R. Samsudin, and A. Shabri, "Exchange Rate Forecasting Using Modified Empirical Mode Decomposition and Least Squares Support Vector Machine," Int J Adv. Soft Compu Appl, vol. 8, no. 3, 2016.

[20] N. I. A. Rashid, A. Shabri, and R. Samsudin, "COMPARISON BETWEEN MEMD-LSSVM AND MEMDARIMA IN FORECASTING EXCHANGE RATE,” J. Theor. Appl. Inf. Technol., vol. 95, no. 2, p. 328, 2017.

[21] J. Nayak, B. Naik, and H. S. Behera, "Fuzzy C-Means (FCM) Clustering Algorithm: A Decade Review from 2000 to 2014," in Computational Intelligence in Data Mining - Volume 2, 2015, pp. 133-149.

[22] T. B. McKee, N. J. Doesken, J. Kleist, and others, "The relationship of drought frequency and duration to time scales," in Proceedings of the 8th Conference on Applied Climatology, 1993, vol. 17, pp. 179-183.

[23] A. K. Mishra and V. R. Desai, "Drought forecasting using feed-forward recursive neural network," Ecol. Model., vol. 198, no. 1-2, pp. 127-138, Sep. 2006.

[24] A. K. Mishra and V. R. Desai, "Drought Forecasting Using Stochastic Models," Stoch. Environ. Res. Risk Assess., vol. 19, no. 5, pp. 326-339, 2005.

[25] M. Shafie-khah, M. P. Moghaddam, and M. K. Sheikh-El-Eslami, "Price forecasting of day-ahead electricity markets using a hybrid forecast method," Energy Convers. Manag., vol. 52, no. 5, pp. 2165-2169, May 2011.

[26] J. Wang and J. Hu, "A robust combination approach for short-term wind speed forecasting and analysis Combination of the ARIMA (Autoregressive Integrated Moving Average), ELM (Extreme Learning Machine), SVM (Support Vector Machine) and LSSVM (Least Square SVM) forecasts using a GPR (Gaussian Process Regression) model," Energy, vol. 93, Part 1, pp. 41-56, Dec. 2015.

[27] J. Gilles and K. Heal, "A parameterless scale-space approach to find meaningful modes in histograms Application to image and spectrum segmentation," Int. J. Wavelets Multiresolution Inf. Process., vol. 12, no. 06, p. 1450044, Oct. 2014.

[28] M. R. Ferreira and F. D. A. De Carvalho, "Kernel fuzzy c-means with automatic variable weighting," Fuzzy Sets Syst., vol. 237, pp. 1-46, 2014.

[29] G. E. Box and G. M. Jenkins, "Time series analysis, control, and forecasting," San Franc. CA Holden Day, vol. 3226, no. 3228, p. 10, 1976.

[30] H. Akaike, "A new look at the statistical model identification," IEEE Trans. Autom. Control, vol. 19, no. 6, pp. 716-723, Dec. 1974. 